\title{
ПРОСТРАНСТВЕННАЯ СВЯЗЬ
}

\section{ИНАЕКСА ПОТРЕБИТЕАЬСКИХ ЦЕН И РАВНОВЕСНОСТИ РЕГИОНААЬНЫХ PЫHKOB ТOBAPOB}

\section{В.М. Тимирьянова}

Тимирьянова Венера Маратовна - кандидат экономических наук, доцент, кафедра экономики и управления на предприятиях торговли и общественного питания. Уфимский институт (филиал) РЭУ им. Г.В. Плеханова, ул. Менделеева, 177/3, Уфа, Россия, 450080. E-mail: 79174073127@mail.ru.

ORCID: 0000-0002-1004-0722

Аннотация. Основой настоящего исследования является авторский подход, предполагающий наличие равновесности регионального розничного рынка товаров, учитывающей определенный баланс внутри групп продавцов и потребителей многообразных товаров. Таким образом, на равновесность регионального розничного рынка товаров будет оказывать влияние наличие следующих «возмущений»: динамика числа предприятий торговли, транспортных и финансовых организаций. Это позволяет выдвинуть гипотезу о существовании связи между пространственным изменением индекса потребительских цен (обобщающей характеристики ценообразования на рынках отдельных потребительских товаров) с равновесностью регионального розничного рынка товаров. Проведенный анализ равновесности розничных рынков товаров субъектов Приволжского федерального округа за 2006-2015 гг. позволил определить, что равновесность может быть достигнута при различных состояниях регионального розничного рынка товаров. С помощью методов пространственной эконометрики построена модель с пространственным лагом, позволяющая протестировать сформулированную гипотезу. Построенная модель подтверждает, что индекс потребительских цен пространственно зависим, а включенный в модель фактор равновесности регионального рынка потребительских товаров влияет на индекс потребительских цен в регионе. Модель может быть улучшена путем учета в ней параметра «равновесность соседних территорий».

Ключевые слова: равновесность, пространственная связь, индекс потребительских цен, региональный розничный рынок товаров, Приволжский федеральный округ

Для ичитирования: Тимирьянова В.M. Пространственная связь индекса потребительских цен и равновесности региональных рынков товаров // Пространственная экономика. 2017. № 2. C. 95-109. DOI: $10.14530 /$ se.2017.2.095-109.

For citation: Timiryanova V.M. Spatial Relation of the Consumer Price Index and the Equilibrium of Regional Goods Markets. Prostranstvennaya Ekonomika = Spatial Economics, 2017, no. 2, pp. 95-109. DOI: 10.14530/se.2017.2.095-109. (In Russian).

(C) Тимирьянова B.М., 2017 


\section{ВВЕДЕНИЕ}

Региональный розничный рынок товаров и его пространственная организация характеризуются динамичностью. Рынок, находящийся в состоянии статического равновесия или стабильного сбалансированного роста, идеальная ситуация. Наблюдаемые реальные процессы позволяют говорить о том, что розничный рынок товаров может достаточно динамично реагировать на отдельные внешние и внутренние факторы, но при этом находиться в равновесном состоянии.

Проблемы рыночного равновесия находятся в фокусе отечественных и зарубежных исследований (Поддубный, Сухорева, 2006; Hamphrey, 1992). Однако рыночное равновесие как соотношение между спросом и предложением на отдельные товары не позволяет раскрыть основы равновесности всего регионального розничного рынка товаров, так как это более широкое понятие, учитывающее определенный баланс внутри групп продавцов и потребителей многообразных товаров. В свою очередь в теории общего равновесия региональный розничный рынок товаров рассматривается только как часть целого.

Равновесность рынка как системы определяется его способностью обеспечивать равновесие различных противоположно направленных сил. Обращаясь к пространственному аспекту равновесности, можно отметить, что неравновесность одних региональных рынков практически всегда отражается на других. Это касается не только взаимосвязи рынков отдельных товаров, но и взаимосвязи рынков, отдельных территорий. Учитывая неоднородность экономического пространства России и сокращение пространственных разрывов в результате НТП, одни и те же факторы могут по-разному влиять на равновесность региональных розничных рынков товаров. Все эти изменения отражаются на индексе потребительских цен, в результате наблюдается беспрерывный в пространстве и времени процесс поиска равновесной цены.

Эта особенность проявляется и в рамках пространственного анализа равновесия, который, как правило, проводится с целью изучения межстранового различия цен на отдельные группы товаров (Takayama, Labys, 1986).

Общепризнанного метода оценки равновесности регионального рынка как системы рынков различных товаров нет, а следовательно, и исследований пространственного взаимодействия этих региональных рынков. В свою очередь рынки товаров взаимозависимы: потребители и производители постоянно решают задачу оптимального выбора, отказываясь от производства/ потребления одних товаров в пользу других. И под влиянием НРП их решения становятся все менее ограниченными территориально, что все сильнее определяет пространственную корреляцию цен. 
Так как региональный рынок товаров представляет собой совокупность многочисленных рынков товаров отдельной территории, в оценке его равновесности предлагается отойти от общепризнанного соотношения спроса и предложения на отдельные товары в сторону оценки взаимодействия субъектов рынка, формирующих спрос и предложение на рынке и инфраструктуры рынка. Это позволяет выдвинуть гипотезу о существовании связи между пространственным изменением индекса потребительских цен (обобщающей характеристики ценообразования на рынках отдельных потребительских товаров) и равновесностью регионального розничного рынка товаров.

\section{ОЦЕНКА РАВНОВЕСНОСТИ РЕГИОНАЛЬНОГО РОЗНИЧНОГО РЫНКА ТОВАРОВ}

Ежедневно можно наблюдать достижение определенного равновесия, результата совокупности сделок множества продавцов и покупателей, нашедших в единицу времени определенное оптимальное, с их точки зрения, решение, связанное с покупкой/продажей товаров. В силу того, что «диалектика рынка состоит в том, что путь к равновесию - цепь непрерывных колебаний, на которые оказывают сильное влияние вектор развития, его интенсивность и масштабы» (Скопина, 2005), а динамическое равновесие отражает «способность единого экономического пространства возвращаться в состояние нового равновесия с новыми параметрами, отличающимися от исходных, после того как оно было выведено из этого состояния под влиянием внешних и внутренних воздействий» (Урунов, 2014), небольшие изменения, свойственные любому динамическому процессу, хотя в определенной степени выбивают систему из равновесия, в данном случае могут рассматриваться как неотъемлемое условие равновесного развития регионального розничного рынка товаров.

Таким образом, рассматривая розничный рынок товаров как систему, встроенную в хозяйственный комплекс региона, можно предположить, что равновесность регионального рынка:

- многократно достигается в условиях его постоянного динамического изменения в определенном промежутке времени;

- определяется множественностью равновесных состояний в разрезе субъектов и объектов регионального рынка потребительских товаров;

- не всегда может быть установлена по совокупному объему спроса (доходы населения) и предложения (объем производства и импорта).

Следовательно, равновесность регионального розничного рынка товаров это характеристика его способности под влиянием внешних и внутренних изменений достигать равновесия в определенном промежутке времени. Так как 
пытаться охватить все группы товаров, реализуемых на современных рынках, практически невозможно, а судить о равновесности рынка по совокупному спросу и предложению необъективно, то возникает потребность в ином методе оценки равновесности регионального розничного рынка товаров. Если рыночное равновесие определяется соотношением спроса и предложения, то равновесность розничного рынка товаров, по мнению автора, связана с субъектами, формирующими спрос и предложение на рынке, инфраструктурой рынка. Остановимся на показателях, которые необходимо учитывать при оценке равновесности регионального розничного рынка товаров.

Спрос падает в случае снижения числа потребителей. В этой ситуации рыночная власть покупателей возрастает, а конкуренция предприятий за платежеспособный спрос усиливается. Предложение формируется предприятиями. Следовательно, о равновесности можно судить по динамике числа покупателей и продавцов. Так как в условиях укрупнения торговых предприятий и развития сетей изменение числа предприятий может сопровождаться неоднонаправленным изменением числа точек реализации, а на розничном рынке товаров торговые точки, принадлежащие одной сети, рассматриваются как конкуренты, то о равновесности регионального розничного рынка товаров можно судить по структурному сдвигу, в расчете которого следует использовать показатель «количество объектов розничной торговли»:

$$
C C_{j}=\frac{T_{o m_{j}}}{T H_{j}},
$$

где $C C_{j}$ - структурный сдвиг в составе участников рынка (объектов розничной торговли и покупателей) в $j$-регионе; $T_{0} m_{j}$ - темп роста количества объектов розничной торговли в $j$-регионе; $T H_{j}-$ темп роста численности населения в $j$-регионе.

Данный показатель, отражающий изменение числа объектов розничной торговли, приходящихся на душу населения, по сути, указывает на «возмущение», произошедшее в отрезке времени. Таким образом, рассматриваемые показатели позволяют говорить только о том, было ли «возмущение» (т. е. некоторая потеря равновесия).

Равновесие потребителя в экономической теории определяется через сопоставление кривой безразличия и бюджетной линии. Изменение доходов (реальных доходов) ведет к изменению спроса: его объема, структуры и пр. Эти изменения, безусловно, ведут к потере равновесия, требуя от предприятий соответствующего преобразования структуры предложения, и сопровождаются изменениями в обороте розничной торговли. Таким образом, при оценке равновесности розничного рынка товаров также необходимо учитывать показатель «темп роста реальных доходов». Так как изменение 
в доходах отдельных групп населения может происходить неравномерно, в дополнение к данному показателю следует включить абсолютный прирост коэффициента Джини, показывающего уровень отклонения от линии равномерного распределения текущего объема распределения доходов населения.

Изменение доходов населения может приводить не только к росту потребления, но и к увеличению накопления или появлению спроса, реализация которого, по мнению потребителя, целесообразнее на другом рынке (например, рынке другой страны). В этом случае структура спроса на региональном розничном рынке товаров сохранится. В свою очередь индекс физического объема оборота розничной торговли позволяет однозначно говорить об изменении потребления. При этом в случае сохранения реальных доходов на прежнем уровне можно говорить об изменении в составе потребителей и структуре их потребления.

Отмеченных выше показателей совершенно недостаточно, чтобы судить о равновесности регионального рынка. Даже при нулевом изменении показателей реальные располагаемые денежные доходы в процентах к предыдущему году и индекса физического объема оборота розничной торговли на рынке могут происходить сдвиги. Чтобы четко понимать, происходит ли изменение продаж, необходимо анализировать сдвиги в товарной структуре оборота розничной торговли. Структурно-динамический анализ может проводиться путем расчета индекса Рябцева (Региональная статистика, 2001). Разработанная им шкала оценки меры существенности структурных различий позволяет оценивать происходящие изменения.

Значительное влияние на равновесность регионального рынка потребительских товаров оказывает его инфраструктура. В общем виде инфраструктура регионального розничного рынка товаров - это сложная организационно-экономическая система, обеспечивающая материальные, финансовые и информационные связи между хозяйствующими субъектами рынка. Поэтому изменение в инфраструктуре рынка неминуемо скажется на рыночных процессах. Об этом свидетельствует, напрмер, уровень чувствительности субъектов рынка потребительских товаров (предприятий обрабатывающей промышленности, торговых предприятий) к изменениям в транспортном секторе (Ефимова, 2009). Таким образом, на равновесность регионального розничного рынка товаров будет оказывать влияние наличие следующих «возмущений»: динамика числа предприятий торговли, транспортных и финансовых организаций.

Для оценки равновесности регионального розничного рынка товаров наиболее целесообразно использовать индикативный метод. В соответствии с ним диагностику равновесности регионального розничного рынка товаров предлагается проводить по показателям, представленным в таблице 1 , интегрированным в единый показатель на основе арифметической средней. 
№ 22017

Таблича 1

Оценка факторов равновесного развития регионального розничного рынка товаров

\begin{tabular}{|c|c|c|c|c|}
\hline \multirow{2}{*}{ Наименование показателя } & \multicolumn{4}{|c|}{ Баллы } \\
\hline & 1 & 2 & 3 & 4 \\
\hline $\begin{array}{l}\text { Структурный сдвиг в составе участников рынка } \\
\text { (объектов розничной торговли и покупателей) (CС) }\end{array}$ & $\begin{array}{r}0,981- \\
1,02\end{array}$ & \begin{tabular}{|l|}
$0,951-098$ \\
$1,021-1,05$ \\
\end{tabular} & $\begin{array}{c}0,901-0,95 \\
1,051-1,1\end{array}$ & $\begin{array}{l}\text { менее } 0,9 \\
\text { более } 1,1\end{array}$ \\
\hline Реальные денежные доходы населения & $\begin{array}{c}99,1- \\
103 \\
\end{array}$ & \begin{tabular}{|c|}
$95,1-99$ \\
$103,1-107$ \\
\end{tabular} & \begin{tabular}{|c|}
$90,1-95$ \\
$107,1-115$ \\
\end{tabular} & $\begin{array}{l}\text { менее } 90 \\
\text { более } 115\end{array}$ \\
\hline $\begin{array}{l}\text { Структурный сдвиг в обороте розничной торговли } \\
\text { отдельных групп «продавцов» (рыночной торговли, } \\
\text { сетевых структур и других форм) (ИДОРТ) }\end{array}$ & & & & \\
\hline иДОРТ $_{j}=\sqrt{\frac{\sum_{i=1}^{n}\left(\text { ДОРТ }_{\text {тек } i j}-\text { ДОРТ }_{\text {баз } i j}\right)^{2}}{\sum_{i=1}^{n}\left(\text { ДОРТ }_{\text {тек } i j}+\text { ДОРТ }_{\text {баз } i j}\right)^{2}}}$, & $\begin{array}{c}\text { менее } \\
0,03\end{array}$ & $0,031-0,07$ & $0,071-0,15$ & $\begin{array}{l}\text { более } \\
0,15\end{array}$ \\
\hline 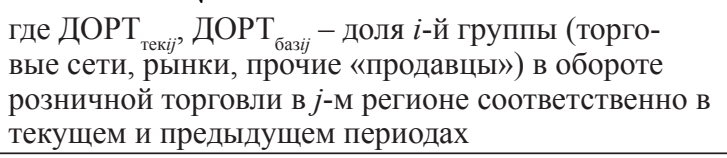 & & & & \\
\hline $\begin{array}{l}\text { Индекс физического объема оборота розничной } \\
\text { торговли }\end{array}$ & $\begin{array}{l}99- \\
103 \\
\end{array}$ & $\begin{array}{c}96,1-99 \\
103,1-106 \\
\end{array}$ & \begin{tabular}{|c|}
$90,1-96$ \\
$106,1-112$ \\
\end{tabular} & $\begin{array}{r}\text { менее } 90 \\
\text { более } 112 \\
\end{array}$ \\
\hline $\begin{array}{l}\text { Прирост коэффициента Джини (индекса концентра- } \\
\text { ции доходов) по модулю }\end{array}$ & $\begin{array}{r}\text { менее } \\
0,003 \\
\end{array}$ & $\begin{array}{c}0,0031- \\
0,007\end{array}$ & $\begin{array}{c}0,0071- \\
0,012 \\
\end{array}$ & $\begin{array}{l}\text { более } \\
0,012 \\
\end{array}$ \\
\hline $\begin{array}{l}\text { Сдвиг в распределении населения по величине } \\
\text { среднедушевых денежных доходов (РВСДД) }\end{array}$ & & & & \\
\hline РВСДД $_{j}=\sqrt{\frac{\sum_{i=1}^{n}\left(Д \Pi_{\text {тек } i j}-\text { ДП баз } i j_{j}\right)^{2}}{\sum_{i=1}^{n}\left(Д \Pi_{\text {тек } i j}+Д_{\text {баз } i j}\right)^{2}}}$ & $\begin{array}{c}\text { менее } \\
0,03\end{array}$ & $0,031-0,07$ & $0,071-0,15$ & $\begin{array}{c}\text { более } \\
0,15\end{array}$ \\
\hline $\begin{array}{l}\text { где ДП текі, }, \text { ДП базіj }_{\text {оро процент людей, имевших доход, }} \text { относящийся К } i \text {-й группе в } j \text {-м регионе соответ- } \\
\text { ственно в текущем и предыдущем периодах }\end{array}$ & & & & \\
\hline $\begin{array}{l}\text { Сдвиги в товарной структуре оборота розничной } \\
\text { торговли (СТСОРТ), \% }\end{array}$ & & & & \\
\hline $\mathrm{CTCOРТ}_{j}=\sqrt{\frac{\sum_{i=1}^{n}\left(И Ф О Р \Pi_{\text {текij }}-100\right)^{2}}{n}}$ & $\begin{array}{c}\text { менее } \\
15\end{array}$ & $15,1-30$ & $30,1-45$ & более 45 \\
\hline $\begin{array}{l}\text { где ИФОРП текі }- \text { индекс физического объема рознич- } \\
\text { ной продажи } i \text {-й группы товара в } j \text {-м регионе, } \% \text {; } \\
n-\text { число групп товаров }\end{array}$ & & & & \\
\hline Темп роста числа предприятий торговли, \% & $\begin{array}{l}99- \\
101 \\
\end{array}$ & $\begin{array}{c}97,1-99 \\
101,1-104 \\
\end{array}$ & $\begin{array}{c}93,1-97 \\
104,1-108 \\
\end{array}$ & $\begin{array}{l}\text { менее } 93 \\
\text { более } 108 \\
\end{array}$ \\
\hline Темп роста числа транспортных организаций, \% & $\begin{array}{l}99- \\
101\end{array}$ & $\begin{array}{c}97,1-99 \\
101,1-104 \\
\end{array}$ & $\begin{array}{c}93,1-97 \\
104,1-108 \\
\end{array}$ & $\begin{array}{l}\text { менее } 93 \\
\text { более } 108\end{array}$ \\
\hline Темп роста числа финансовых организаций, \% & $\begin{array}{l}99- \\
101\end{array}$ & $\begin{array}{c}97,1-99 \\
101,1-104 \\
\end{array}$ & \begin{tabular}{|c|}
$93,1-97$ \\
$104,1-108$ \\
\end{tabular} & \begin{tabular}{|l|} 
менее 93 \\
более 108 \\
\end{tabular} \\
\hline
\end{tabular}

Примечание. 1, 2, 3, 4 - баллы, которые присваиваются показателю при достижении определенного значения на основе экспертных оценок. Для показателей ИДОРТ и РВСДД баллы проставлялись на основе шкалы оценки меры существенности структурных различий, предложенной Рябцевым (Региональная статистика, 2001). 
Уровень и характер проявления изменений в состоянии регионального розничного рынка товаров оцениваются при сравнении фактических (текущих) значений индикаторов с их пороговыми (критериальными) значениями.

Региональные розничные рынки товаров предлагается разбить на четыре группы в зависимости от полученного значения (в баллах):

- равновесный (менее 2,0);

- умеренно равновесный $(2,0-2,49)$;

- умеренно неравновесный $(2,5-2,99)$;

- неравновесный (3,0 и более).

По рассмотренным выше показателям была проведена оценка равновесности развития рынка потребительских товаров Приволжского федерального округа (табл. 2). В качестве основных источников информации были использованы материалы официальной статистики (Единая..., 2017).

Согласно представленным данным, незначительное, но «возмущение» на рынке присутствует всегда. Рынки потребительских товаров отдельных регионов под воздействием различных факторов смещались с одного равновесного состояния в другое.

Таблиц̧а 2

Оценка равновесности региональных рынков потребительских товаров территорий ПФО

\begin{tabular}{l|c|c|c|c|c|c|c|c|c|c}
\hline \multicolumn{1}{c|}{ Территория } & 2006 & 2007 & 2008 & 2009 & 2010 & 2011 & 2012 & 2013 & 2014 & 2015 \\
\hline Республика Башкортостан & 2,6 & 2,8 & 2,6 & 1,9 & 2,0 & 2,1 & 2,4 & 2,3 & 1,5 & 2,2 \\
\hline Республика Марий Эл & 2,8 & 2,7 & 2,6 & 1,9 & 2,0 & 2,1 & 2,2 & 2,4 & 2,3 & 2,2 \\
\hline Республика Мордовия & 2,5 & 2,5 & 3,2 & 2,1 & 2,4 & 1,8 & 2,0 & 2,4 & 2,2 & 2,6 \\
\hline Республика Татарстан & 2,5 & 2,9 & 2,6 & 2,1 & 2,5 & 2,2 & 2,8 & 2,1 & 2,3 & 2,6 \\
\hline Удмуртская республика & 2,9 & 2,7 & 2,3 & 2,2 & 2,4 & 2,2 & 2,2 & 2,1 & 2,2 & 2,4 \\
\hline Чувашская республика & 3,5 & 2,8 & 2,6 & 1,9 & 2,1 & 2,0 & 2,7 & 2,2 & 2,0 & 2,2 \\
\hline Пермский край & 2,7 & 2,5 & 2,6 & 2,4 & 1,9 & 2,0 & 1,8 & 1,9 & 1,6 & 2,3 \\
\hline Кировская область & 2,8 & 3,0 & 3,1 & 2,2 & 2,5 & 2,7 & 2,1 & 1,9 & 1,8 & 2,5 \\
\hline Нижегородская область & 2,8 & 2,4 & 2,4 & 2,1 & 2,2 & 2,1 & 2,4 & 1,9 & 2,2 & 2,5 \\
\hline Оренбургская область & 2,8 & 2,5 & 3,0 & 2,1 & 2,0 & 1,8 & 1,8 & 1,9 & 1,6 & 2,1 \\
\hline Пензенская область & 2,7 & 3,1 & 2,5 & 2,6 & 2,0 & 2,1 & 1,9 & 2,1 & 1,6 & 2,2 \\
\hline Самарская область & 2,5 & 2,7 & 2,3 & 2,6 & 2,1 & 2,0 & 2,1 & 2,2 & 2,5 & 2,5 \\
\hline Саратовская область & 2,7 & 2,3 & 2,6 & 2,0 & 2,7 & 2,3 & 2,1 & 1,9 & 1,8 & 2,3 \\
\hline Ульяновская область & 3,0 & 2,9 & 2,5 & 2,0 & 2,3 & 2,3 & 2,2 & 1,7 & 1,8 & 2,8 \\
\hline
\end{tabular}

Примечание: данные рассчитаны на основе подхода, изложенного в таблице 1.

Источник: рассчитано по: Единая..., 2017.

Сравнение данных 2006-2008 гг. и 2010-2014 гг. позволяет говорить о том, что региональные рынки потребительских товаров в 2007 г. были более изменчивыми. В первую очередь нужно отметить изменения со стороны 
спроса. Спровоцированное ростом денежных доходов (РДД), перераспределение населения по величине среднедушевых денежных доходов привело к изменению как объема спроса (ИФОРТ), так и его структуры (СТСОРТ). Равновесность на рынках достигалась не только ростом цен, но и увеличением со стороны предложения, о чем свидетельствует показатель «структурный сдвиг» (СС), указывающий на увеличение в 2007 г. числа предприятий. Индекс физического объема оборота розничной торговли в 2007 г. варьировался от 109 до 127 в разрезе регионов. Определенное влияние на равновесность оказывало изменение товарных потоков (сокращение числа оптовых предприятий в одних регионах и увеличение в других). Увеличение объема рынка сопровождалось увеличением числа предприятий, обслуживающих товарно-денежные потоки (транспортных организаций и финансовых учреждений). В 2011 г. отмечается более равновесное развитие региональных рынков потребительских товаров. Сдвиги как со стороны предложения, так и со стороны спроса слабее, нежели в 2007 г. В 2014 г. наблюдается выход из равновесного состояния только в нескольких субъектах РФ в ПФО, в 2015 г. наблюдается переход в неравновесное состояние в республиках Марий Эл и Мордовии, Ульяновской области. Из общей картины выбивается только Пермский край, находящийся практически все последние годы в условиях равновесности. При этом только для данного края среди территорий ПФО характерна высокая рентабельность в обрабатывающей промышленности.

Для оценки тесноты связи показателей и выделения общих и частных региональных тенденций развития рынка потребительских товаров был проведен корреляционный анализ. Он позволил выделить показатели, динамика которых носит общий характер для всех рассматриваемых региональных рынков потребительских товаров. Так, однонаправленными являются динамика реальных денежных доходов населения региональных потребительских рынков, индекс физического объема оборота розничной торговли, распределение населения по величине среднедушевых денежных доходов. Однако ряд регионов имеет свои особенности, что проявляется в различии в структуре предприятий, в развитии инфраструктуры. Так, на конец 2010 г. оборот розничной торговли розничных торговых сетей в процентах к общему обороту розничной торговли, по данным Федеральной службы государственной статистики в Республике Башкортостан, составлял 9,2\%, а в Нижегородской области - 19\%, в 2015 г. - 13,5 и 24,9\% соответственно. В Марий Эл к 2015 г. достиг 35,6\%. В ряде территорий изменялась инфраструктура рынка. Во всех территориях наблюдались сдвиги в товарной структуре оборота розничной торговли.

Анализ данных в динамике позволяет говорить о том, что равновесность может быть достигнута при различных состояниях регионального рынка 
потребительских товаров. Так, в 2011-2014 гг. равновесными были рынки Оренбургской и Пермской областей. Однако в Оренбургской области индекс цен колебался от 104,5 до 111,57\%, рентабельность в обрабатывающем производстве от $-1,39$ до $3,4 \%$, рентабельность в розничной торговле от $-4,62$ до $1,28 \%$. В то время как в Пермской области индекс цен на товары колебался от 106,2 до $110,88 \%$, рентабельность в обрабатывающем производстве от 17,67 до $24,97 \%$, рентабельность в розничной торговле от 0,44 до $1,28 \%$.

Понимая, что рост рынка потребительских товаров возможен только при условии изменения среды и выхода из равновесного состояния, необходимо опасаться длительного пребывания в условиях равновесности. Анализ данных в динамике показывает, что в рассмотренных субъектах РФ, в которых наблюдается изменение состояния через смену умеренно неравновесного состояния на умеренно равновесное, адаптация к изменениям внешней среды происходит быстрее.

\section{ПРОСТРАНСТВЕННЫЙ АСПЕКТ В РАВНОВЕСНОСТИ РОЗНИЧНОГО РЫНКА ТОВАРОВ}

Региональные розничные рынки товаров не являются закрытыми системами. Изменения на рынке в одном регионе отражаются на соседних. Соответственно нарушение равновесности рынка товаров в одном регионе влияет на равновесность рынков товаров соседних территорий. Это явление до сих пор остается малоизученным. В свою очередь, равновесие между спросом и предложением приводит к формированию равновесной цены, которая также характеризуется пространственной зависимостью, когда цены на одной территории связаны с ценами в соседних территориях, т. е. наблюдается пространственная автокорреляция цен (Файзлыев, 2014; Barrett, Li, 2002; Fackler, Goodwin, 2001).

Если рассматривать равновесность как некоторую характеристику регионального розничного рынка товаров, отражающую отклонение текущего равновесного состояния от базового, то отклонение равновесной цены от базовой будет характеризовать индекс потребительских цен.

Связь между равновесностью и пространственным изменением цен оценивается автором с помощью пространственной регрессионной модели. Расчеты проводились с помощью программы GeoDa (Anselin, 1990; Anselin, Syabri, Kho, 2006), по данным о равновесности и индексе потребительских цен розничных рынков товаров субъектов Приволжского федерального округа в 2006-2015 гг.

Расчеты индекса Морана показали разнонаправленное изменение про- 
странственной автокорреляции равновесности развития розничного рынка потребительских товаров в течение рассматриваемого периода, т. е. в отдельные годы равновесность на региональных рынках потребительских товаров достигалась при неравновесном состоянии его соседей. По индексу потребительских товаров значения наблюдений на соседних территориях являются подобными, индекс Морана положительный.

Для выбора пространственной модели выполнено шесть тестов (табл. 3).

Диагностика пространственной связи переменных

Таблициа 3 для выбранной матрицы смежности регионов

\begin{tabular}{l|c|c|c}
\hline \multicolumn{1}{c|}{ Тест } & MI/DF & Значение & Probability \\
\hline Moran's I (error) & 0,7969 & 14,0738 & 0,00000 \\
\hline Lagrange Multiplier (lag) & 1 & 198,6698 & 0,00000 \\
\hline Robust LM (lag) & 1 & 16,6699 & 0,00004 \\
\hline Lagrange Multiplier (error) & 1 & 183,4000 & 0,00000 \\
\hline Robust LM (error) & 1 & 1,4002 & 0,23669 \\
\hline Lagrange Multiplier (SARMA) & 2 & 200,0700 & 0,00000 \\
\hline
\end{tabular}

Источник: рассчитано по: Единая..., 2017.

Показатель Морана I составил 0,79, что свидетельствует о сильной пространственной автокорреляции остатков. Тесты LM (множитель Лагранжа) показывают высокие значения, что свидетельствует о наличии пространственной зависимости. Эти тесты помогают понять, какой тип пространственных зависимостей лучше использовать. Robust LM (error) несущественен, что говорит о том, что при наличии запаздывающей зависимой переменной зависимость ошибки исчезает. Следовательно, лучше использовать модель пространственного лага (Spatial Lag Model):

$$
y_{i t}=a+b x_{i t}+\rho W y_{i t}+\varepsilon
$$

где $y_{i t}-$ зависимая переменная (индекс потребительских цен в $i$-м регионе в году $t) ; a, b$ - коэффициенты регрессионного уравнения; $x_{i t}$ - факторная переменная (равновесность $i$-го регионального розничного рынка товаров в году $t) ; \rho$ - коэффициент пространственной авторегрессии; $W y_{i t}-$ пространственный лаг эндогенной переменной $y$, определяемый произведением значений зависимой переменной в других регионах в году $t$ и матрицы смежности этих регионов.

Матрица пространственных весов задавалась экзогенно. Ее спецификация является наиболее сложным и спорным вопросом в моделировании пространственных взаимосвязей. В текущем исследовании использовалась матрица смежности регионов: 


$$
w_{i j}=\left\{\begin{array}{c}
1, \text { если регион } i \text { и } j \text { имеют общую границу; } \\
0, \text { если } i=j ; \\
0, \text { если регион } i \text { не имеет общей границы с регионом } j .
\end{array}\right.
$$

В результате нами была построена регрессионная модель (Spatial Lag Model), значения коэффициентов которой представлены в таблице 4.

Таблица 4

Результаты построения регрессионной модели индекса потребительских цен

\begin{tabular}{l|c|c|c|c}
\hline \multicolumn{1}{c|}{ Показатель } & Коэффициент & Стандартная ошибка & z-value & Probability \\
\hline Constant & 12,419 & 2,827 & 4,39 & 0,00001 \\
\hline $\begin{array}{l}\text { W_Индекс } \\
\text { потребительских цен }\end{array}$ & 0,8726 & 0,0267 & 32,7 & 0,00000 \\
\hline Равновесность & 0,5909 & 0,2683 & 2,2 & 0,02767 \\
\hline
\end{tabular}

Источник: рассчитано автором по данным (Единая..., 2017).

По сути в модели увязываются равновесность рынка (определенная по представленной выше методике, в баллах) и индекс цен на товары в соседних территориях. Результаты расчетов показывают положительную пространственную авторегрессию. О качестве модели можно судить по следующим показателям: коэффициент детерминации $\left(\mathrm{R}^{2}\right)=0,885687$; логарифм функции правдоподобия (Log Likelihood) $=-231,337$; информационный критерий Акаике (Akaike Info Criterion) = 468,674; информационный критерий Шварца (Schwarz Criterion $)=477,499$; Sigma-square $=1,15682$; стандартная ошибка регрессии $($ S.E of Regression $)=1,07556$; тест Бройша - Пэгана $($ Breusch-Pagan Test $)=$ 7,2126 (prob. =0,00724); оценка правдоподобия $($ Likelihood Ratio Test $)=233,7889$ $($ prob $=0,00000)$. Оценка по тесту Бройша - Пэгана на гетероскедастичность имеет существенное значение, что говорит о том, что гетероскедастичность является серьезной проблемой. Тест отношения правдоподобия (Likelihood Ratio Test) подтверждают присутствие пространственной авторегрессии.

Полученные результаты позволяют объяснять изменение индекса цен на региональных розничных рынках товаров. Наибольший маржинальный эффект имеет изменение индекса цен в соседних территориях (0,88\%). Изменение равновесности на $1 \%$ ведет к изменению индекса потребительских цен только на 0,1\%. Однако влияние равновесности на индекс цен очевидно, и его нельзя отбрасывать. Например, как отмечалось ранее, в 2015 г. переход в неравновесное состояние наблюдался в трех субъектах ПФО, в том числе в Ульяновской области. Индекс цен на товары в данной области был самым высоким среди субъектов ПФО и составил 115,2\%. При этом сама Ульяновская область окружена субъектами РФ со сравнительно невысоким значением индекса потребительских цен (табл. 5). 
№ 22017

Таблииа 5

Сравнительная характеристика Ульяновской области и Чувашской республики

\begin{tabular}{|c|c|c|}
\hline Наименование & Ульяновская область & Чувашская республика \\
\hline $\begin{array}{l}\text { Индекс цен на товары в } \\
\text { субъекте РФ, \% }\end{array}$ & 115,2 & 112,4 \\
\hline $\begin{array}{l}\text { Индекс цен на товары в со- } \\
\text { седних субъектах РФ, \% }\end{array}$ & $\begin{array}{c}\text { Чувашская республика }-112,4 \\
\text { Республика Мордовия }-112,7 \\
\text { Пензенская область }-112,1 \\
\text { Республика Татарстан }-111,7 \\
\text { Самарская область }-114,8 \\
\text { Саратовская область } 111,9 \\
\text { (среднее значение } 112,7 \text { ) }\end{array}$ & $\begin{array}{c}\text { Республика Мордовия - } 112,7 \\
\text { Нижегородская область - 112,5 } \\
\text { Республика Татарстан - } 111,7 \\
\text { Республика Марий Эл - 113,9 } \\
\text { Ульяновская область }-115,2 \\
\text { (среднее значение } 113,2 \text { ) }\end{array}$ \\
\hline $\begin{array}{l}\text { Равновесность розничного } \\
\text { рынка товаров субъекта РФ }\end{array}$ & $\begin{array}{c}3 \text { балла } \\
\text { (неравновесное состояние) }\end{array}$ & $\begin{array}{c}2,5 \text { балла } \\
\text { (умеренно неравновесное со- } \\
\text { стояние) } \\
\end{array}$ \\
\hline $\begin{array}{l}\text { Темп роста численности } \\
\text { населения / темп роста } \\
\text { количества объектов тор- } \\
\text { говли, \% }\end{array}$ & $99,6 / 101,8$ & $99,8 / 100,8$ \\
\hline $\begin{array}{l}\text { Реальные денежные до- } \\
\text { ходы, \% }\end{array}$ & 90 & 96,8 \\
\hline $\begin{array}{l}\text { Индекс физического объ- } \\
\text { ема оборота розничной } \\
\text { торговли, \% }\end{array}$ & 86,5 & 92 \\
\hline $\begin{array}{l}\text { Прирост коэффициента } \\
\text { Джини (индекса кон- } \\
\text { центрации доходов) по } \\
\text { модулю }\end{array}$ & 0,372 & 0,356 \\
\hline $\begin{array}{l}\text { Сдвиг в распределении } \\
\text { населения по величине } \\
\text { среднедушевых денежных } \\
\text { доходов }\end{array}$ & 0,05 & 0,07 \\
\hline $\begin{array}{l}\text { Сдвиги в товарной струк- } \\
\text { туре оборота розничной } \\
\text { торговли }\end{array}$ & 34 & 26 \\
\hline $\begin{array}{l}\text { Темп роста числа предпри- } \\
\text { ятий торговли, \% }\end{array}$ & 105 & 104 \\
\hline $\begin{array}{l}\text { Темп роста числа транс- } \\
\text { портных организаций, \% }\end{array}$ & 104 & 104 \\
\hline $\begin{array}{l}\text { Темп роста числа финансо- } \\
\text { вых организаций, \% }\end{array}$ & 96 & 98 \\
\hline
\end{tabular}

Неравновесность рынка потребительских товаров оказала негативное влияние на фактическое значение индекса цен на товары в Ульяновской области. В свою очередь в Чувашской республике, несмотря на более высокое значение индекса цен в соседних субъектах РФ, удалось достичь более низкого значения этого показателя.

Построенная модель подтверждает, что индекс потребительских цен пространственно зависим, а включенный в модель фактор равновесности 
регионального рынка потребительских товаров влияет на индекс потребительских цен в регионе. Таким образом, сформулированная выше гипотеза доказана.

\section{ЗАКЛЮЧЕНИЕ}

Региональный розничный рынок товаров можно считать лишь относительно обособленным, что проявляется в динамике отдельных показателей. В данной работе был предложен и реализован подход, позволяющий подойти к вопросу оценки равновесности регионального розничного рынка потребительских товаров. Реализация данного подхода на данных за 2006-2015 гг. позволила получить альтернативные оценки состояния региональных рынков Приволжского федерального округа на основе значений косвенных показателей. Проведенные расчеты также указывают на то, что равновесность на розничном рынке товаров Приволжского федерального округа связана с изменением индекса цен на нем (на формирование равновесной цены).

Используемый автором подход для оценки равновесности может быть улучшен путем включения в него дополнительных (и / или исключения некоторых уже имеющихся) показателей, характеризующих противоположно направленные силы, которые позволили бы получить более точную альтернативную оценку. В результате этих изменений может быть решена выявленная проблема гетероскедастичности, а следовательно, улучшена построенная модель с пространственным лагом, позволяющая прогнозировать изменение потребительских цен.

Проведенный анализ пока не позволяет утверждать, что равновесность имеет четко выраженную пространственную зависимость. Однако в рамках модели с пространственным лагом удалось определить влияние равновесности на пространственное изменение индекса цен. Модель может быть улучшена путем учета в ней равновесности соседних территорий.

\section{СПИСОК ЛИТЕРАТУРЫ}

Единая межведомственная информационно-статистическая система (ЕМИСС) / ФСГС. 2017. URL: http://www.fedstat.ru (дата обращения: апрель 2017).

Eфимова Е.Г. Роль транспорта в экономическом развитии региона: международный аспект // Вестник СПбГУ. Серия 5. Экономика. 2009. Вып. 1. С. 77-85.

Поддубный B.B., Сухарева Е.A. Исследование динамической модели рынка вальрасовского типа со многими товарами // Вестник Томского государственного университета. 2006. № 293. С. 53-58.

Региональная статистика / под ред. В.М. Рябцева, Г.И. Чудилина. М., 2001. 380 с.

Скопина И.В. Оценка тенденций развития, колеблемости и цикличности конкурент- 
ного потребительского рынка // Управление экономическими системами. 2005. № 1. URL: http://www.uecs.ru/marketing/item/12-2011-03-18-12-45-01 (дата обращения: март 2017).

Урунов А.А. Единое и общее экономическое пространство. М.: СИНЕРГИЯ, 2014. 388 с. Файзльев А.P. Математические методы и модели анализа пространственной структуры системы городской торговли: автореф. дисс. ... канд. экон. наук: 08.00.13. Волгоград, 2014. 24 с.

Anselin L. Spatial Dependence and Spatial Structural Instability in Applied Regression Analysis // Journal of Regional Science. 1990. Vol. 30. Issue 2. Pp. 185-207. DOI: 10.1111/j.1467-9787.1990.tb00092.x.

Anselin L., Syabri I., Kho Y. GeoDa: An Introduction to Spatial Data Analysis // Geographical Analysis. 2006. Vol. 38. Issue Pp. 5-22. DOI: 10.1111/j.0016-7363.2005.00671.x.

Barrett Ch.B., Li J.R. Distinguishing between Equilibrium and Integration in Spatial Price Analysis // American Journal of Agricultural Economics. 2002. Vol. 84 (2). Issue 2. Pp. 292-307. DOI: 10.1111/1467-8276.00298.

Fackler P.L., Goodwin B.K. Spatial Price Analysis // Handbook of Agricultural Economics. 2001. Vol. 1. Pp. 972-1024. DOI: 10.1016/s1574-0072(01)10025-3.

Humphrey T.M. Marshallian Cross Diagrams and their Uses Before Alfred Marshall: The Origins of Supply and Demand Geometry // Economic Review. 1992. March/april. Pp. 3-23.

Takayama T., Labys W.C. Spatial Equilibrium Analysis // Handbook of Regional and Urban Economics. 1986. Vol. 1. Pp. 171-198.

\section{SPATIAL RELATION OF THE CONSUMER PRICE INDEX AND THE EQUILIBRIUM OF REGIONAL GOODS MARKETS}

\section{V.M. Timiryanova}

Venera Maratovna Timiryanova - Candidate of Economic Sciences, Assistant Professor, Department of Economics and Management of Trade and food Service Enterprises.

Ufa Institute of Plekhanov Russian University of Economics, 177/3 Mendeleev str., Ufa, Russia, 450080. E-mail: 79174073127@mail.ru.

ORCID: 0000-0002-1004-0722

Abstract. The author of the study makes an assumption about the existence of the equilibrium of the regional retail goods market, that takes into account a certain balance inside the groups of sellers and consumers of various goods. That way the equilibrium of regional retail goods market will be influenced by the following 'disturbances': the dynamics of the number of retailers, transport and financial organizations. This allows to make a hypothesis about the existence of connection between spatial changes of the consumer price index (an indicator of pricing in the markets of certain consumer goods) and the equilibrium of the regional retail goods market. The analysis of equilibrium of the retail goods market among the subjects of Volga Federal District in 2006-2015 allowed determining that equilibrium can be achieved in different states of the regional retail market of goods. Through methods of spatial econometrics the model was built with spatial lag which allows testing the hypothesis. The model confirms that the consumer price index has spatial dependency while the included factor of regional retail market equilibrium influences the consumer price index in the region. The model can be improved by including the equilibrium of neighboring regions.

Keywords: equilibrium, spatial relation, consumer price index, regional retail market of goods, Volga Federal District 
Anselin L. Spatial Dependence and Spatial Structural Instability in Applied Regression Analysis. Journal of Regional Science, 1990, vol. 30, issue 2, pp. 185-207. DOI: 10.1111/j.1467-9787.1990.tb00092.x.

Anselin L., Syabri I., Kho Y. GeoDa: An Introduction to Spatial Data Analysis. Geographical Analysis, 2006, vol. 38, no. 1, pp. 5-22. DOI: 10.1111/j.0016-7363.2005.00671.x.

Barrett Ch.B., Li J.R. Distinguishing between Equilibrium and Integration in Spatial Price Analysis. American Journal of Agricultural Economics, 2002, vol. 84 (2), issue 2, pp. 292-307. DOI: 10.1111/1467-8276.00298.

Efimova E.G. The Role of Transport in the Regional Economic Development: International Aspect. Vestnik SPbGU. Seriya 5. Ekonomika = St. Petersburg University Journal of Economic Studies, 2009, Issue 1, pp. 77-85. (In Russian).

Fackler P.L., Goodwin B.K. Spatial Price Analysis. Handbook of Agricultural Economics, 2001, vol. 1, pp. 972-1024. DOI: 10.1016/s1574-0072(01)10025-3.

Fayzlyev A.R. Mathematical Methods and Models of Analysis of Spatial Structure of Urban System Trade: Abstract of Dissertation of Candidate of Economic Science: 08.00.13. Volgograd, 2014, 24 p. (In Russian).

Humphrey T.M. Marshallian Cross Diagrams and their Uses Before Alfred Marshall: The Origins of Supply and Demand Geometry. Economic Review, 1992, March/April, pp. 3-23.

Poddubny V.V.,Sukhareva E.A. The Research of Dynamic Model of Walrasian Market with Many Goods. Vestnik Tomskogo Gosudarstvennogo Universiteta = Tomsk State University Journal, 2006, no. 293, pp. 53-58. (In Russian).

Regional Statistics. Edited by V.M. Ryabtsev, G.I. Chudilin. Moscow, 2001, 380 p. (In Russian).

Skopina I.V. Evaluation of Development Trends, Variability and Cyclic Nature of the Competitive Consumer Market. Upravlenie Ekonomicheskimi Sistemami = Management of Economic Systems, 2005, no. 1. Available at: http://www.uecs.ru/marketing/item/122011-03-18-12-45-01 (accessed March 2017). (In Russian).

Takayama T., Labys W.C. Spatial Equilibrium Analysis. Handbook of Regional and Urban Economics, 1986, vol. 1, pp. 171-198.

Unified Interdepartmental Information and Statistical System. Federal State Statistics Service. Available at: http://www.fedstat.ru (accessed April 2017). (In Russian).

Urunov A.A. Single and Common Economic Space. Moscow, 2014, 388 p. (In Russian). 